\title{
Storage and retrieval processes in unlearning*
}

\author{
JUDITH A. PETRICH \\ University of Pittsburgh, Pittsburgh, Pennsylvania 15260
}

\begin{abstract}
A revised interference theory interpretation of unlearning is presented which is based on an explicit storage-retrieval model. The theory holds that the first and second lists are stored as separate memory units. Intrusions of first-list items during second-list learning are viewed as allowing the opportunity for dual storage of the first-list item within both memory units. In standard retroactive inhibition experiments in which no special cues for list discrimination are provided, the major factor which produces unlearning is held to be the S's inability to retrieve the first-list memory unit.
\end{abstract}

The purpose of this paper is to present a theory of unlearning based on explicit assumptions about storage and retrieval processes. It should be noted that the term "unlearning" is used here in an empirical sense. Thus, the present theory is concerned with the explanation of retention decrements which occur in the retroactive inhibition paradigm on unpaced retention tests, i.e., under conditions designed to minimize the effects of response competition.

The present theory falls under the general rubric of interference theory in that interlist intrusions are assumed to be of major importance in the retroactive inhibition paradigm. Thus, the theory may be viewed as a revision of current interference theory in that it offers a different framework for the interpretation of familiar concepts such as associative unlearning and response-set interference.

The primary reason for formulating a revision of interference theory is, obviously, to attempt to provide a more adequate explanation of unlearning phenomena. In its present form, the theory proposed in this paper serves this function in two ways. First, the theory offers explanations for two empirical phenomena which, as Postman and Underwood (1973) note, create difficulties for current forms of interference theory. Specifically, the present theory is based on a new assumption about the effect of interlist intrusions which enables the theory to explain both the fact that unlearning is never complete and the fact that the degree of unlearning is not directly related to the frequency of interlist intrusions. Second, the storage and retrieval assumptions of the present theory provide a means of incorporating item-specific and list-related processes within one integrated conceptual framework. Thus, the present theory offers a potential resolution of a current theoretical controversy.

The paper is organized into three main sections. The first section includes the presentation of the theory

\footnotetext{
*Preparation of this paper was supported in part by Grant GB-28682 from the National Science Foundation. The author would like to thank James Voss and James Pellegrino for their helpful suggestions during the preparation of this manuscript. Thanks are also due Harry Chiesi, $\mathbf{R}$ obert Berger, and Cindy Thomas for their assistance in gathering the data reported in this paper.
}

along with a discussion of the empirical and logical bases for the major assumptions of the theory. The second section includes a comparison of the present theory with current forms of interference theory. The third section presents an evaluation of the theory in terms of existing data.

\section{A THEORY OF STORAGE AND RETRIEVAL PROCESSES IN UNLEARNING}

For purposes of clarity, it should be noted that the following discussion of the theory deals primarily with the A-B, A-C (same stimuli, different unrelated responses) and the $A-B, A-B_{R}$ (same stimuli, same responses, re-paired) paradigms only. Furthermore, the present discussion assumes standard experimental conditions in which the experimental context remains essentially constant and the same type of material is used in the first and second lists. This emphasis is justifiable in that the negative transfer paradigms (A-C and $A \cdot B_{R}$ ) have been the ones most extensively investigated in unlearning research and the retention decrement for first-list pairs observed in the standard retroactive inhibition paradigm is the basic phenomenon to be explained. However, discussion of the A-B, D-C (unrelated pairs) paradigm and of specific phenomena observed under "nonstandard" conditions are incorporated into the discussion where appropriate.

The general frame of reference for the theory may be stated in terms of two basic assumptions. First, it is assumed that a paired-associate (PA) list may be treated as a higher order memory unit. In other words, the outcome of learning a circumscribed unchanging set of pairs is considered to be a unit analogous to a category in the sense that the pairs "belong together" as a group even though there may be no strong interpair associations or particular orderings within this group. In other words, it is assumed that the $\mathrm{S}$ in a paired-associate learning task learns a list, not just a number of independent pairs. Second, it is assumed that retrieval is a two-step process involving retrieval of the memory unit or list followed by retrieval of pairs within the list. List retrieval is considered to be of major importance in 
multiple-list situations such as the retroactive inhibition paradigm.

Within the general framework, the first basic assumption about storage embodied within the present theory is that the first and second lists in a transfer paradigm are represented in memory as separate units regardless of stimulus similarity. In the $A-C$ and $A-B_{R}$ paradigms, there are two obvious ways to conceptualize the storage of the two lists. One conceptualization would hold that the second-list responses are at tached to the stimulus traces that were established during first-list learning. This view suggests the formation of only one memory unit, which incorporates both lists and within which the different responses learned to the same stimulus are stored in the form of a response hierarchy. Thus, addressing one representation of a stimulus would allow $S$ to retrieve both responses learned to that stimulus. The alternative conceptualization would hold that, when the " $A$ " stimuli are presented in the second list, they are shunted to a new location in memory, so that the first- and second-list pairs are stored in separate memory units. Thus, in order to retrieve both responses associated with a particular stimulus, $S$ would have to address two separate representations of the same stimulus in memory. The present theory assumes that the latter alternative represents the appropriate model for storage processes in transfer situations.

While this assumption of separate storage necessarily implies that more than one representation of the same stimulus is stored in the A-C and $A \cdot B_{R}$ paradigms, it should be noted that this assumption does not necessarily imply changes in the encodings of the stimuli. Instead of assuming that separation of the lists occurs as a function of changes in the perceptual or semantic encoding of the stimulus items per se, it is assumed that this separation is based on list characteristics such as changes in list content in the A-C paradigm and the temporal position of the list in the transfer sequence in both the $A-C$ and $A-B_{R}$ paradigms. In other words, in terms of the present theory, identical nominal stimuli could be encoded in the same way in both lists, but the two representations should be distinguishable on the basis of list membership or "locus."

The most important aspect of the separate storage assumption is the assertion that, at the end of second-list learning, there are two separate memory units rather than one. This assertion is of central importance to the present theory, but taken by itself it would imply that $S$ must retrieve the first-list memory unit in order to recall any first-list pairs. An important qualification is placed on this implication, however, by the second major storage assumption of the present theory. Specifically, it is assumed that interlist intrusions may occur during second-list learning and that such intrusions provide an opportunity to store the intruding first-list responses within the second-list memory unit.

Interlist intrusions are assumed to occur because the formation of the second-list memory unit occurs in the context of an already well established first-list memory unit. Since the learning of a PA list is a gradual process, at least in the sense that not all of the pairs are learned immediately, it seems to be only reasonable to assume that the formation or stabilization of a memory unit corresponding to the list is also a gradual process. Thus, while the second-list memory unit is being formed, it is quite possible that a response will occasionally be selected from the wrong memory unit. According to the present theory, the outcome of this interaction between the memory units is that some first-list responses will be stored within both memory units. Thus, if $S$ retrieves the second-list memory unit on a retention test, he will be able to retrieve not only the second-list responses but also those first-list responses which intruded. On the other hand, if $S$ should be able to retrieve only the first-list memory unit, he would be able to recall only first-list responses.

This discussion of the interaction between memory units leads to one of the most important hypotheses derived from the present theory. Contrary to other forms of interference theory, the present theory predicts that interlist intrusions will facilitate recall of the pairs which intrude. Data relevant to this hypothesis will be considered after a discussion of the logical and empirical bases for the assumption of separate storage.

The logical basis for the separate storage assumption is an analysis of the usual experimental procedure used in retroactive inhibition experiments. Procedurally, the first and second lists are separated by a brief instruction interval, during which $S$ is informed that he is now going to learn a new, different list of pairs. Thus, the E defines the second list as a separate task, both by the procedure used (introducing a break) and by the instructions given. If this procedure has any effect on $\mathrm{S}$, it should bias him toward "starting over" to form a new memory unit for the second list.

The empirical basis for this assumption rests on an experiment by Postman and Stark (1963). Using the A-B, A-C paradigm, these investigators attempted to manipulate S's strategy during second-list learning by instructing him prior to the second list about the eventual recall test for the first test. The effect of these instructing him prior to the second list about the eventual recall test for the first list. The effect of these self-generated responses were subsequently compared with free association norms, Postman and Stark (1963) found that most of the normative responses used were associates of the stimulus items only, i.e., $S$ did not attempt to mediate by using an associate of the first-list response. These data provide some support for the assumption of separate storage in that S's strategy in this situation seemed to be to maintain distinct first and second lists.

The second major storage assumption, that interlist intrusions allow for dual storage of the first-list pair, and the hypothesis that interlist intrusions will facilitate recall are also based on both logical and empirical considerations. Logically, it is obvious that the 
elicitation of a first-list response during second-list learning provides an opportunity to rehearse that first-list pair despite the fact that such elicitations may interfere with learning the second-list pair. In fact, it could be argued that this rehearsal property of intrusions has been virtually ignored only because interference theorists have been primarily concerned with explaining retention decrements and, consequently, attention has been focused on the interference potential of intrusions.

Empirically, the present hypothesis receives indirect support from experiments designed to test the ability to acquire and maintain two responses to the same stimulus concurrently. Experiments on probability learning (Popp \& Voss, 1965) indicate that $S$ can acquire two responses to the same stimulus concurrently, while, in the usual A-B, A-C paradigm, Postman and Parker (1970) have shown that $S$ can maintain the first-list response during second-list learning if asked to give both responses on test trials. These experiments provide indirect support for the present hypothesis because they indicate that the acquisition of a new response to a given stimulus does not necessarily require the suppression of existing associations to that stimulus. Thus, these data suggest that it would, in fact, be possible for Ss to maintain rather than suppress first-list responses which intruded during second-list learning.

Data more directly relevant to the present hypothesis are found in experiments designed to determine the relationship between frequency of overt intrusions and unlearning (Keppel \& Rauch, 1966; Paul \& Silverstein, 1968). The data from these experiments are contradictory in that Keppel and Rauch (1966) found that increasing the frequency of overt interlist intrusions during second-list learning had no effect upon unlearning, while Paul and Silverstein (1968) found that such increases produced less unlearning. Superficially, then, the Keppel and Rauch (1966) data provide little support for either the traditional elicitation hypothesis or for the present revision of that hypothesis, while the data from Paul and Silverstein's (1968) experiment support the present hypothesis. However, a closer look at these experiments suggests two conclusions: First, the Paul and Silverstein (1968) experiment may represent a more adequate test of the relationship between intrusions (overt + covert) and unlearning, and second, neither experiment really addresses the most important questions about intrusions.

The first conclusion is based on the fact that the experimental procedure used by Paul and Silverstein (1968) probably increased the total number of interlist intrusions, both overt and covert, while the procedure used by Keppel and Rauch (1966) may not have. Keppel and Rauch (1966) increased the frequency of overt interlist intrusions by instructing $S$ to give the response from the first-list pair during second-list learning whenever he could not remember the second-list response. Essentially, these instructions ask $\mathrm{S}$ to replace omission errors with first-list responses. To the extent that some omission errors in second-list learning under uninstructed conditions result from covert response competition, these instructions may have changed only the ratio of overt to covert interference without altering the total amount of interference. If this is the case, then neither the traditional elicitation hypothesis nor the present hypothesis would predict any change in amount of unlearning.

Paul and Silverstein (1968), on the other hand, attempted to increase the frequency of overt interlist intrusions by having Ss learn a mixed list of A-B and A-C pairs as the second list; i.e., they attempted to inhibit the development of list differentiation. On the common assumption that degree of interlist interference is inversely related to list differentiation (cf. Postman \& Underwood, 1973), this procedure should have increased the total frequency of interlist intrusions, both overt and covert. Thus, since unlearning is presumably related to total interlist interference rather than to overt interference only, the Paul and Silverstein (1968) experiment provides the more adequate test of the relationship between interlist interference and unlearning.

Although the two experiments just cited do provide some support for the present hypothesis, neither experiment addresses the basic questions about intrusion processes: Do interlist intrusions occur without either special instructions to give first-list responses or unusual lists and, if so, do such intrusions exhibit any reliable relationship with retention performance? In an attempt to provide some objective evidence relevant to these points, two experiments have been performed in our laboratory. The first experiment was exploratory in the sense that it was carried out to determine whether $\mathbf{S}$ would, in fact, report errors that they thought of when asked to do so. The data from this experiment indicated that the procedure was useful with regard to providing objective data on interlist intrusions. Therefore, the second experiment was performed both to replicate the effects observed in Experiment I and to investigate more adequately the relationship to recall performance of both item strength and intrusions.

\section{EXPERIMENT I}

\section{Method}

Design and Procedure. All $\mathrm{Ss}$ in this experiment learned two $\mathrm{CVC}$ adjective lists in the A-B, A-C transfer paradigm and were then given a written MMFR test for the two lists. Each list consisted of eight pairs. The first list was learned to a criterion of one perfect trial; 10 trials were given on the second list.

During both stages of learning, the study-test procedure was used, with a 2-sec presentation rate on study trials and S-paced test trials. On each test trial, S was given a blank sheet of paper and instructed to copy each stimulus as it was presented, to write beside it any responses which occurred to him that he believed to be incorrect, to say the correct response aloud if he knew it, and to call for the next stimulus when he was ready. 
Although our main interest was in the occurrence of interlist intrusions during $\mathrm{A}-\mathrm{C}$ learning, Ss recorded errors during both stages of learning both to acquaint them with the procedure and to mask the specific purpose of the experiment. Basically, this procedure represents a direct attempt to observe intrusions without any specific instructions to $S$ about what to record.

Subjects. Fourteen volunteers from undergraduate courses at the University of Pittsburgh served as Ss in the experiment.

\section{Results}

During second-list learning, an average of 20.21 $(S D=9.50)$ errors per $S$ were recorded and, of these, $55 \%$ were interlist intrusions. Thus, the mean number of interlist intrusions reported was $11.28(\mathrm{SD}=8.06)$ per $\mathrm{S}$. These intrusions did not represent mere repetitions of the same troublesome item, as the mean number of different first-list responses which intruded at least once was $4.50(\mathrm{SD}=1.84)$ out of 8 possible. Finally, it is interesting to note that $54 \%$ of the interlist intrusions occurred along with the correct second-list response, i.e., $S$ wrote the first-list response and also recalled the correct second-list response.

In order to test the specific prediction that first-list items which intruded during second-list learning would tend to be recalled, a correlated $t$ test was performed which compared the proportion recalled for items which intruded appropriately at least once with the proportion recalled for items which never intruded appropriately. First-list responses which were elicited only by inappropriate stimuli during second-list learning were not classified as intrusions for purposes of these tests, because such mispaired intrusions would not be expected to facilitate recall. If it is assumed that a correctly paired intrusion may produce storage of the correct first-list pair within the second-list memory unit, then it must also be assumed that consistent production of a mispaired intrusion may result in storage of an inappropriate first-list pair within the second-list memory unit. This is especially likely to occur, since $S$ receives no feedback regarding first-list pairs during second-list learning.

The correlated $t$ test indicated that items which intruded appropriately were more likely to be recalled than items which did not $[\mathrm{t}(13)=3.25, \mathrm{p}<.01]$. The mean proportion of appropriate intrusions recalled was $0.77(\mathrm{SD}=0.23)$; the mean for the remaining items was $0.51(\mathrm{SD}=0.35)$.

In order to determine whether this facilitation effect depended on the strength of the first-list item at the end of first-list learning, the items were divided into strong and weak categories by determining the median proportion correct responses for all items during first-list learning and classifying items above the median $(0.69)$ as strong and those below as weak. ${ }^{1}$ The proportion of items recalled from each of these four categories is presented in the first row of Table 1. Although there is a tendency for intrusions to be recalled more often than nonintrusions in both strength categories, correlated $t$ tests indicated that this tendency was significant only for strong items $[t(8)=3.49, p<.01]$. [For weak items, $\mathrm{t}(11)=1.27, \mathrm{p}>.05$.$] Thus, the data from this$ experiment suggested that intrusions facilitate recall, but that this facilitation might depend on item strength. The second experiment was designed to investigate the reliability of these findings.

\section{EXPERIMENT II}

\section{Method}

Design and Procedure. This experiment involved two conditions: an experimental condition in which Ss learned the two CVC adjective lists in the A-B, A-C paradigm and a retroactive inhibition control condition in which $S$ s worked on a filler task (math problem) in place of second-list learning.

In this experiment, the strength of the first-list pairs was manipulated by varying the number of presentations for separate sets of pairs within a list. First-list learning consisted of six study-test trials on a list of 12 pairs. However, 6 of the 12 pairs, designated "strong," were presented on all six trials, while the remaining 6 pairs, designated "weak," were presented on only two of the six trials. The set of weak pairs was divided into three subsets of two pairs. One of these subsets was presented and tested on Trials 1 and 4, the second subset on Trials 2 and 5, and the third on Trials 3 and 6 . Thus, on each trial only 8 of the 12 pairs were actually presented.

A ppropriate counterbalancing procedures were employed so that each of the two lists was used equally often as the first list in each condition and each set of six pairs within a given list served equally often as "strong" and "weak" pairs.

The procedure with regard to study and test trials was the same as that used in Experiment $I$. The Ss in both the experimental and control conditions reported errors during first-list learning. After the six trials on the first list, Ss in the experimental condition received eight trials on the 12-pair A-C list. Ss in the control condition worked on arithmetic problems for $30 \mathrm{~min}$. In a third stage of the experiment, all Ss were given an unpaced MFR test for the first-list pairs.

Subjects. The Ss were volunteers from undergraduate psychology courses at the University of Pittsburgh. There were $16 \mathrm{~S} s$ in each of the two conditions.

Table 1

Mean Proportion Recalled on the MFR Test as a Function of Item Strength and Intrusions During Second-List Learning

\begin{tabular}{|c|c|c|c|c|c|c|c|c|}
\hline & \multicolumn{8}{|c|}{ Item Category } \\
\hline & \multicolumn{2}{|c|}{ Strong-Intrusion } & \multicolumn{2}{|c|}{ Strong-Nonintrusion } & \multicolumn{2}{|c|}{ Weak-Intrusion } & \multicolumn{2}{|c|}{ Weak-Nomintrusion } \\
\hline & Mean & $\mathrm{SD}$ & Mean & SD & Mean & SD & Mean & $\mathrm{SD}$ \\
\hline Experiment I & 0.86 & 0.28 & 0.55 & 0.34 & 0.53 & 0.40 & 0.38 & 0.43 \\
\hline Experiment II & 0.60 & 0.31 & 0.32 & 0.32 & 0.50 & 0.46 & 0.21 & 0.25 \\
\hline
\end{tabular}




\section{Results}

Since the strong and weak pairs were not tested an equal number of times, first-list performance was assessed in terms of the proportion of test trials for each set of items on which a correct response occurred. These data were analyzed by means of a 2 by 2 by 32 (conditions by strength by Ss) analysis of variance, with conditions as a between-S variable and strength as a within-S variable. The only significant effect in this analysis was the effect of strength $[F(1,30)=56.00$, $\mathrm{p}<.001]$. The mean proportion correct responses for strong items was $0.65(\mathrm{SD}=0.18)$; for weak items the mean was $0.47(S D=0.20)$. Thus, as expected, varying the presentation frequency of the items in the list did result in differential performance, while the two groups of Ss did not differ in performance on the first list overall.

Since there were a number of items for which no correct responses were given during first-list learning, an additional analysis was performed to compare the number of items for which at least one correct response occurred in the experimental and control conditions. In this analysis also, the only significant effect was the main effect of item strength $[F(1,30)=38.55$, $\mathrm{p}<.001$ ]. The mean number of strong items responded to correctly was $5.68(\mathrm{SD}=0.63)$; the corresponding mean for weak items was $4.28(\mathrm{SD}=1.54)$.

Two analyses were also performed on the MFR data. The first analysis was performed on the number of correct responses for each $\mathrm{S}$; the second analysis was performed on the proportion of "learned" items that were recalled, where an item was classified as "learned" if it had been responded to correctly at least once during first-list learning. The two analyses produced parallel results with the main effects for both condition $[F(1,30)$ $=21.16$ and 41.39 , respectively, $\mathrm{p}<.001]$ and strength $[F(1,30)=30.57$ and 8.55 , respectively, $p<.01$ being significant $]$. The interaction between these variables was not significant in either analysis $[F(1,30)=1.91$ and 0.64 , respectively, $p>.05]$. Thus, significant unlearning did occur, with mean recall for the control condition being $8.44(\mathrm{SD}=2.42)$ items compared with a mean of $4.50(\mathrm{SD}=2.26)$ for the experimental condition. In addition, strong items were more likely to be recalled in both conditions, with a mean of $4.09(\mathrm{SD}=1.77)$ strong items recalled vs a mean of $2.38(\mathrm{SD}=1.76)$ for weak items.

It should be noted at this point that none of the items with zero correct responses during first-list learning ever intruded during second-list learning, nor were any of these items ever recalled correctly. Therefore, these items were ignored in subsequent analyses.

The data of major interest in this experiment, of course, are the data on interlist intrusions. During second-list learning, an average of $32.31(\mathrm{SD}=3.08)$ errors per $\mathrm{S}$ were recorded and $51 \%$ of these were interlist intrusions. Thus, the mean number of interlist intrusions reported per $S$ was $16.56(S D=10.66)$. In terms of the number of different first-list responses which intruded at least once, the mean was 5.38 $(\mathrm{SD}=2.12)$ items. As in the first experiment, a large proportion of the interlist intrusions (32\%) occurred along with the correct second-list response.

The effects of strength and of the occurrence of intrusions on recall were assessed by means of a 2 by 2 by 10 (intrusions by strength by Ss) analysis of variance, with both intrusions and strength as within-S variables. As in Experiment $I$, first-list pairs were classified as intrusions if the first-list response was elicited at least once by the appropriate stimulus during second-list learning. This analysis was performed on the proportion of learned items recalled in each strength-intrusion category. Six Ss had to be excluded from this analysis because they contributed no data to one of the four cells. For three of these Ss, no weak items intruded; for two of them, all strong items intruded; and for the remaining $S$, no strong items intruded.

The only significant effect in this analysis was the main effect of intrusions $[F(1,9)=20.09, p<.01]$. The interaction of Strength by Intrusion Variables was not significant, $F<1$. The mean proportion of intruding items recalled was $0.54(\mathrm{SD}=0.40)$, while the mean proportion of nonintruding items recalled was 0.21 $(\mathrm{SD}=0.25)$.

The same conclusions about the relative effects of strength and intrusions are also supported by separate correlated t tests for each variable in which all Ss can be included: for the strength variable, $\mathrm{t}(15)=1.44, \mathrm{p}>.05$; for the intrusion variable, $\mathrm{t}(15)=2.90, \mathrm{p}<.02$.

For purposes of comparison with Experiment I, mean proportion recalled per strength-intrusion category is presented in the second row of Table 1. Although the overall level of recall is lower in Experiment II, it is obvious that intrusions tended to facilitate recall for all items. The lower level of recall in this experiment is most likely attributable to the fact that Ss had a longer list to learn and were not given the opportunity to learn the list to criterion.

The data from the present experiments offer strong support for the hypothesis that the elicitation of a first-list response by the stimulus with which it was paired during first-list learning will facilitate subsequent recall of that pair. In addition, the data from the second experiment suggest that the intrusion history of an item is a more important factor in recall than the original strength of the item and that intrusions facilitate recall regardless of strength.

With regard to mispaired intrusions, it was noted earlier that inappropriate first-list pairings might be stored within the second-list memory unit if a stimulus tended to elicit only inappropriate first-list responses. There are not many cases in which this occurred; $6 \%$ of the stimuli in Experiment $I$ and 12\% in Experiment II 
elicited only inappropriate responses. Nevertheless, retention performance as a function of the type of interlist errors elicited during second-list learning is worth looking at because the acquisition or stabilization of inappropriate pairings of first-list items may be relevant to the explanation of the small retention decrements observed on recognition tests in the A-B, A-C paradigm (e.g., McGovern, 1964). Therefore, the stimuli in both experiments were classified into four categories based on the type of interlist errors elicited during second-list learning: appropriate intrusions only, both appropriate and inappropriate intrusions; inappropriate intrusions only; and none. The proportion of items recalled for the stimuli in each category is presented in Table 2. The rank order of these categories is identical in the two experiments and the data at least suggest that, just as appropriate intrusions facilitate retention, mispaired intrusions may inhibit subsequent retention of the correct first-list pair.

Consideration of the hypothesis that intrusions will facilitate recall leads rather directly into a discussion of the retrieval assumptions of the theory because this facilitation hypothesis derives from the assumption that only the second-list memory unit will be retrieved on recall tests given immediately after second-list learning. Thus, on an immediate recall test, the present theory suggests that only those first-list pairs which were stored within the second-list memory unit will be recalled. The rationale behind the assumption that the first-list memory unit is inaccessible on immediate recall tests will now be considered.

As noted previously, the present theory holds that retrieval in a multilist task is a two-step process involving retrieval of the list followed by retrieval of pairs within the list. Successful completion of the first step in this process, list retrieval, depends upon the presence of an adequate list retrieval cue.' A list retrieval cue may be defined as any general characteristic of the list material or of the experimental situation which could be used to characterize a particular list and, consequently, could serve to elicit the appropriate memory unit on a retention test.

In multilist situations, an adequate list retrieval cue obviously must be discriminative so that the appropriate list can be retrieved. The present theory suggests that, under standard retroactive inhibition conditions, the

Table 2

Proportion of Items Recalled in MFR as a Function of the Type of Interlist Errors Elicited by the Stimulus in Second-List Learning

\begin{tabular}{lccc}
\hline & \multicolumn{2}{c}{ Experiment } & \\
\cline { 2 - 3 } & I & II & $\begin{array}{c}\text { Com- } \\
\text { bined }\end{array}$ \\
\hline Appropriate Only & 0.81 & 0.66 & 0.73 \\
Appropriate + Inappropriate & 0.69 & 0.48 & 0.55 \\
Inappropriate Only & 0.17 & 0.21 & 0.20 \\
None & 0.54 & 0.35 & 0.44 \\
\hline
\end{tabular}

first-list memory unit will be inaccessible on immediate retention tests because there is no discriminative list retrieval cue for the first list, while potential list retrieval cues common to both lists converge on the second list.

In the usual retroactive inhibition experiment, there are only two obvious types of cues provided by the experimental situation which could serve as list retrieval cues: the experimental context and the temporal ordering of the lists. Since it seems to be likely that cues based on temporal order would be used in the form of labels such as "first" and "second," cues based on temporal order will be termed "labeling cues" in this discussion.

With regard to the first-list memory unit, it is suggested that the only list retrieval cue directly attached to this unit is the experimental context. The most important aspect of this statement is the assumption that there is no "first-list" cue directly at tached to the first-list memory unit. This assumption is based on the fact that the $S$ has no reason to label pairs as "first-list" pairs during first-list learning because he does not know that he will learn a second list.

There are two implications of the preceding assumption that should be noted. First, this assumption implies that there is a difference between an "absolute" and a "relative" or discriminative cue. In other words, there is a difference between "This is the first PA list I learned today," when that list may also be the only list to be learned, and "This is the first of two PA lists that I learned." Second, the assumption implies that an objective fact that could serve as a list retrieval cue, such as the temporal priority of the first list in a transfer paradigm, must be actively processed in some way if it is to be effective. Thus, it is suggested that the objective temporal position of the first list may not be translated into an effective discriminative retrieval cue, because during learning $S$ does not know that this aspect of the situation is potentially important.

Some support for the preceding viewpoint can be gleaned from Winograd's (1968) monograph on list discrimination, in which he noted that, if Ss were told before list presentation that they would be asked to identify list membership, they adopted special strategies such as refusing to attend to the second list or adding mnemonic tags to items. Although we have no data to indicate that such strategies are actually necessary to provide a list retrieval cue for the first-list memory unit, the implication is clear that Ss who have no prior knowledge about the subsequent stages of the experiment are less likely to actively develop cues for list membership. Thus, under standard retroactive inhibition procedures, it seems to be entirely reasonable to assume that labeling cues, if they are used at all, begin to develop only during second-list learning. At this point, the status of the first list as the first of two or more lists is defined, but it is now too late to attach the label "first" to the first-list memory unit because there is no 
distinguishable characteristic of that list to which the cue can be attached. Therefore, it seems to be likely that only those first-list items which intrude during second-list learning will be appropriately labeled. On the other hand, the status of the second list as a second list is defined from. the outset in learning, so that the $\mathrm{S}$ can label all of the second-list pairs while they are being learned. This should allow the labeling cues to become an effective list retrieval cue for the second-list memory unit.

According to this viewpoint, at the end of second-list learning, the only retrieval cue for the first-list memory unit is the experimental context. However, contextual cues are more recently and, given that high degrees of second-list learning are common in unlearning experiments, perhaps more strongly associated with the second-list memory unit. Therefore, the dominance of the second-list memory unit might be expected on this basis alone. To the extent that labeling cues are important as list retrieval cues, these cues should also tend to elicit the second-list memory unit, since these cues should be operative only during second-list learning. In fact, even instructions to recall the first list might lead to retrieval from the second-list memory unit, because this unit presumably contains the only appropriately labeled first-list pairs.

The basic retrieval assumption of the present theory, then, is that the first-list memory unit is available, but inaccessible, on immediate recall tests and that retrieval is based on the underlying second-list memory unit. One way to test the assumption that retrieval is based on a single memory unit which contains hierarchically stored items is to investigate the order in which $S$ retrieves responses when he is asked to recall both lists. A hierarchical storage hypothesis obviously predicts that the $S$ should tend to recall both responses to the same stimulus consecutively. This prediction has been supported in several experiments (Martin \& Mackay, 1970; Pellegrino, 1972; Petrich, 1973). Thus, there is evidence to support the notion that first-list items are retrieved from a memory unit which includes both firstand second-list responses. However, two important questions remain to be answered. First, is it the case that items $S$ fails to recall are not stored in this memory unit, i.e., is hierarchical storage within the second-list memory unit responsible for retrieval, as the present theory suggests? Second, does a separate inaccessible first-list memory unit actually exist or is the memory unit sampled at recall actually the only one $S$ formed? In other words, is the assumption that $S$ does not add second-list responses to the existing first-list memory unit correct?

To summarize, the present theory provides the following description of the processes that occur in the retroactive inhibition paradigm: (1) During first-list learning, a memory unit is formed which can be retrieved on the basis of contextual cues. (2) During second-list learning, a new memory unit is formed, but some of the first-list responses may intrude during the formation of this unit. These intruding responses may be stored within the second-list memory unit. (3) During second-list learning, the second-list memory unit becomes attached to the contextual cues and labeling cues may be used by $\mathrm{S}$ for both second-list responses and for those first-list responses which intrude. (4) On an immediate recall test, $S$ will tend to retrieve the second-list memory unit, and only those first-list responses that have been stored within it will be retrieved.

According to the present theory, then, one of the major factors involved in the production of unlearning is the inaccessibility of the first-list memory unit. This conceptualization obviously implies that unlearning should be reduced if an adequate list retrieval cue for the first-list memory unit is available to $\mathrm{S}$. Thus, the present theory would explain the findings of less unlearning in the A-B, D-C than in the A-C or A-B $B_{R}$ paradigms (e.g., McGovern, 1964) and of less unlearning in the A-C paradigm when the $\mathrm{B}$ and $\mathrm{C}$ responses are drawn from different form classes (e.g., Postman, Keppel, \& Stark, 1965) on the basis of list retrieval cues.

In the D-C paradigm, the first-list stimuli should provide access to the first-list memory unit on the retention test because these items were presented only during first-list learning. In the different form-class condition, a distinctive, easily labeled at tribute of the first-list is provided which should enable $S$ to retrieve the first-list memory unit.

Despite the fact that these findings are in accord with the present theory as presented to this point, the fact that unlearning is reduced but not eliminated in these situations indicates that inaccessibility of the first-list memory unit by itself does not account completely for unlearning. Therefore, the present theory must include mechanisms to account for the fact that retrieval of the first-list memory unit does not enable $S$ to retrieve all of the pairs stored within that unit.

Within the structure of the present theory, the most likely hypothesis to account for retention decrements when the first-list memory unit is accessible is that some form of competition occurs between the two memory units. Sufficient conditions are present for competition to occur because the variables under consideration may increase the accessibility of the first-list memory unit, but these variables should not decrease the accessibility of the second-list memory unit. Therefore, in the situations under consideration, both memory units are active simultaneously and retention decrements may be produced by output interference (cf. Postman, Stark, \& Henschel, 1969; Postman \& Underwood, 1973).

Although it is not possible to specify the exact nature of this output interference process at the present time, data consistent with such an interpretation can be presented. Empirically, output interference refers to the 
fact that probability of recall for the remaining items in a list tends to decrease as the number of items already recalled increases. Accordingly, if an output interference explanation is appropriate in the D-C paradigm and in the different form-class condition, we would expect to find dominance of the second-list memory unit on recall tests, i.e., $\mathrm{S}$ should tend both to separate the lists in recall and to recall the second list first.

In the case of the D-C paradigm, Postman, Stark, and Henschel (1969) have reported exactly these effects, i.e., on a recall test given immediately after D-C learning, Ss tended to recall the second-list pairs first. In addition, these investigators found that this dominance of the second list tended to decrease on a delayed retention test, while first-list recall increased. Thus, the expected relationship between priority of the second list in recall and performance on the first list was observed.

Since the present theory suggests that the same type of interference is occurring in the different form-class condition as in the D-C paradigm, we investigated organization of recall as a function of form-class similarity in the A-B, A-C paradigm. The purpose of this experiment was to determine whether the tendencies to organize recall by lists and to recall the second list first would occur in the different form-class condition.

\section{EXPERIMENT III}

\section{Method}

Design and Procedure. Three conditions were included in this experiment, two experimental conditions, in which A-B, A-C lists were learned, and a retroactive inhibition control condition, in which a filler task (math problems) was substituted for second-list learning. For all Ss the first list consisted of eight CVC adjective pairs. For Ss in the AC-same condition, the responses in the second list were adjectives unrelated to those used in the first list. For Ss in the AC-different condition, the second-list responses were two-digit numbers.

The PA study-test procedure was used during learning. The first list was learned to a criterion of one perfect trial; 10 trials were presented on the second list. The items were presented via Carousel projector on both study and test trials at a 2 -sec rate, with a 2-sec interval between study and test trials and a 4-sec interval between test and study trials. Ss in the control condition worked on the filler task for $7 \mathrm{~min}$ following first-list learning.

Immediately after second-list learning, or filler task completion, all Ss were given a free-recall test for responses. A free-recall test was used because this procedure provides the least biased estimate of S's organizational tendencies. Ss in the experimental conditions were instructed to recall the responses from both lists in any order; Ss in the control condition, of course, recalled only first-list responses.

The two CVC adjective lists constructed for the AC-same condition were used equally often as the first list in each condition. Two separate sets of number responses were also constructed and used equally often as the second-list responses in the AC-different condition.

Subjects. The Ss were volunteers from the introductory psychology course at the University of Pittsburgh, who served in the experiment in partial fulfillment of a course requirement. A total of $30 \mathrm{Ss}$ were tested, providing an $\mathrm{N}$ of 10 per condition.
}

\section{Results}

In a 3 by 2 (conditions by lists) analysis of variance on trials to criterion in first-list learning, there were no significant effects. A 2 by 2 (conditions by lists) analysis of correct responses in second-list learning for the experimental conditions also indicated no significant effects.

Since there was no indication that the lists used differed in difficulty during learning, a one-way analysis of variance was performed on the number of first-list responses that were recalled correctly on the free-recall test. This analysis indicated a significant difference among conditions $[F(2,27)=12.17, p<.01]$. Mean correct responses for the three conditions are presented in Table 3. A Dunnett's test indicated that significant unlearning occurred in both A-C conditions $[\mathrm{t}(27)=$ 3.33 for AC different and 8.64 for AC same, $p<.01$ in both cases]. In addition, a t test comparing the two experimental conditions indicated that recall in the AC-different condition was significantly superior to recall in the $\mathrm{AC}$-same condition $[\mathrm{t}(18)=5.13, \mathrm{p}<.01]$. Thus, the effect of form-class similarity was replicated in the present experiment.

The primary purpose of the experiment was to determine whether Ss in the AC-different condition would organize their recall by list membership and whether they would tend to recall the second-list responses first. Scores for clustering by list were computed using the standard score formula proposed by Hudson and Dunn (1969). The mean of the list clustering scores in the AC-different condition was 2.70 $(\mathrm{SD}=0.64)$, which was significantly different from zero $[\mathrm{t}(9)=12.86, \mathrm{p}<.001]$. All of the Ss in this condition showed a strong tendency to organize by lists, with 8 of the 10 Ss showing complete separation of the lists. By contrast, the mean list clustering score in the AC-same condition was $0.26(\mathrm{SD}=1.54, \mathrm{t}<1)$, and only 1 of the 10 Ss completely separated the lists.

The relative index of priority (RIP), proposed by Flores and Brown (1974), was used to assess the tendency to recall the second-list responses first in the AC-different condition. This score varies from -1 to +1 . where, in the present situation, -1 would indicate complete priority of the first list and +1 would indicate complete priority of the second list. The mean RIP score for the $10 \mathrm{Ss}$ in the AC-different condition was t0.50 $(S D=0.78)$. Since it was hypothesized that the second list would be dominant on the recall test, the significance of the mean RIP score was assessed by means of a one-tailed $t$ test which indicated that the mean did differ significantly from zero $[\mathrm{t}(9)=1.92$. $\mathrm{p}<.05$ ]. However, this overall mean actually underestimates the strength of the dominance effec1 because it includes two Ss who showed complete dominance of the first list, while eight of the Ss showed the expected priority effect for the second list, with six 
of them having scores of +1 . The mean RIP score for these eight Ss was $+0.87 \quad(S D=0.20)$, which is significantly greater than zero $[\mathrm{t}(7)=12.43, \mathrm{p}<.001]$. Thus, there is evidence from both the D-C paradigm and the different form-class condition which suggests that the second-list memory unit is not only available but tends to be dominant in these conditions immediately after second-list learning. Therefore, the notion that retention decrements in these conditions may be a function of competition between memory units or output interference seems to be reasonable and the remaining problem is to specify the exact nature of this interference effect.

Before closing this discussion of retrieval processes, there is one final variable that has been investigated in unlearning research which should be considered, namely, type of retention test. When the unlearning effect in the $\mathrm{A}-\mathrm{C}$ and $\mathrm{A}-\mathrm{B}_{\mathrm{R}}$ paradigms is assessed by both recall and recognition tests, three effects have been observed that we wish to consider. First, in the A-C paradigm, less unlearning occurs on recognition than on recall tests. Second, the unlearning effect observed in the A-C paradigm on recognition tests is small, but often statistically reliable. Third, the unlearning effect observed in the $A-B_{R}$ paradigm remains relatively constant regardless of the type of test. These effects were initially reported by McGovern (1964).

Within the present theory, the effects observed in the A-C paradigm may be explained in terms of list retrieval processes along with the hypothesis of dual storage of some first-list pairs. On the one hand, presentation of all first-list items on a recognition test should enable Ss to retrieve the first-list memory unit because the set of items which was presented only during first-list learning (the responses) is made available on the test and some of the items are stored only in the first-list memory unit. In a sense, $E$ is presenting the best possible list retrieval cue-the list itself. Given access to the first-list memory unit, $S$ should be able to perform correctly on some pairs that would be unavailable on a recall test, because they had not been stored within the second-list memory unit.

On the other hand, given that some of the first-list responses are stored in both memory units, presentation of only first-list items on a recognition test does not preclude arousal of the second-list memory unit. Therefore, the small retention decrement that is observed on recognition tests in the A-C paradigm may be due to the fact that both memory units are aroused. However, the interference produced by the arousal of the second-list memory unit cannot be attributed to output interference in the case of recognition tests because $S$ does not have to produce any of the responses. Nevertheless, the simultaneous activation of both memory units could influence performance by introducing some disorganization into the retrieval process if $S$ tends to use both memory units on the test.
Table 3

Mean Correct Responses on the Free Recall Test as a Function of Condition

\begin{tabular}{lcc}
\hline Condition & Mean & SD \\
\hline Control & 6.40 & 0.66 \\
AC-Different & 5.10 & 2.21 \\
AC-Same & 3.10 & 0.94 \\
\hline
\end{tabular}

If the second-list memory unit is addressed for a certain stimulus, an error could result either because that first-list pair was not stored within the second-list memory unit or because an inappropriate first-list response has been stored with that stimulus during second-list learning.

With regard to the $A-B_{R}$ paradigm, it has been demonstrated that retention performance remains relatively constant in this condition, regardless of the type of test. In essence, the A-C and $A-B_{R}$ paradigms produce approximately equivalent degrees of unlearning on recall tests, but performance in the A-C paradigm is superior to performance in the $A-B_{R}$ paradigm on recognition tests (McGovern, 1964; Postman \& Stark, 1969). The present theory suggests that this difference between the two paradigms is attributable to list retrieval processes. In other words, it is assumed that both the $A-C$ and $A-B_{R}$ paradigms involve separate storage of the lists, with dual storage of intruding first-list responses. The major difference between the paradigms is assumed to reside in the fact that it is much more difficult to provide access to the first-list memory unit in the $A-B_{R}$ paradigm. Recognition tests do not provide such access because none of the list items belongs uniquely to the first list. Thus, in the $A-B_{R}$ paradigm, $S$ will presumably retrieve only the second-list memory unit on either type of test and, consequently, only those first-list responses that were stored within the second-list memory unit will be retrieved on either type of test.

\section{RELATIONSHIP TO EXISTING THEORIES}

The present theory is an item-specific theory in the sense that the fate of any item depends upon the intrusion history of that item. However, the present theory also incorporates list-level processes such as inaccessibility of the first-list memory unit and output interference. Therefore, as noted at the outset, the present theory provides a framework for the integration of different viewpoints within interference theory. The purpose of this section of the paper is to deal explicitly with the similarities and differences between the present theory and previous conceptualizations of unlearning.

The present theory resembles extinction theory (e.g., McGovern, 1964) in that the fate of each first-list item is assumed to depend upon its intrusion history. However, there are two fundamental differences between extinction theory and the present theory. First, within 
extinction theory, interlist intrusions are assumed to have only negative consequences for retention of the first-list pair, while the present theory holds that interlist intrusions have a positive function by allowing $\mathrm{S}$ to store some first-list responses within the memory unit which will be sampled at recall. Second, extinction theory implies that there is only one memory unit formed when identical stimuli are present in both lists, while the present theory holds that the first and second lists are stored separately even though some first-list responses will be stored within both memory units. Thus, the concept of list retrieval has no logical place within extinction theory, whereas this concept is of central importance for the present theory.

While the storage assumptions of the present theory separate it from extinction theory, these assumptions also produce some similarity between the present theory and the hypothesis of response-set interference (Postman \& Stark, 1969; Postman, Stark, \& Fraser, 1968). The two theories are similar in that both assume suppression of the first-list memory unit during second-list learning because of the increasing dominance of the second-list memory unit. However, there are differences in the conceptualization of what is being suppressed and how. First, the hypothesis of response-set interference holds that the set of first-list responses is being suppressed, while the present theory holds that the memory unit which was formed during first-list learning and which contains the first-list pairs is becoming inaccessible. Second, the hypothesis of response-set interference emphasizes the development of internal criteria for responding as the basis of second-list dominance, while the present theory emphasizes both internal (labeling) and external (contextual) cues. The fundamental difference between the two theories, however, concerns the role of interlist intrusions and the outcome of the inaccessibility of the first-list memory unit. The hypothesis of response-set interference assumes that interference from any first-list response will tend to increase the restrictiveness of the selection criteria and the corresponding suppression of all first-list responses. The present theory assumes that the dominance of the second-list memory unit occurs primarily because of the simple fact that the second list is learned in the same context as the first list and because $S$ does not know that he must develop discriminative cues until he begins to learn the second list. Within the present theory, then, interlist intrusions affect the retrievability of the responses which intrude but are not directly responsible for the inaccessibility of the first-list memory unit. Furthermore, since the hypothesis of response-set interference states that the entire set of first-list responses is suppressed, this theory provides no ready explanation for the fact that some first-list responses are available for recall. The present theory, on the other hand, by providing for dual storage of the first-list pairs, offers an explicit explanation for the fact that some first-list pairs can be retrieved in spite of the inaccessibility of the first-list memory unit.

Before turning to a consideration of the ability of the present theory to explain existing data, note should be taken of Bower's (1972) recent discussion of "list markers" in multilist PA situations, as this concept may be confused with the concept of list retrieval cues used in the present theory. Bower (1972) seems to be primarily concerned with the possibility that each individual stimulus in a PA list may become associated with internal contextual elements, so that the nominal stimulus-context compound is the effective stimulus for each response. Although some of the contextual elements may be associated with more than one stimulus-thus providing for list identification-others are presumably unique to each stimulus. Thus, when a given stimulus is presented, retrieval of the correct response depends upon the ability of the stimulus to redintegrate the "list marker." The present concept of list retrieval cues differs from Bower's (1972) concept in a number of respects. First, we are concerned with more general cues (e.g., "first list," "the list I learned in this room"), analogous to category labels, that can arouse an entire list structure. Second, the formation of these cues is viewed in the present theory as being less automatic and more purposeful than in Bower's (1972) theory. Bower implies that the elaboration of the PA stimulus in terms of contextual elements is a necessary part of learning, whereas the present theory suggests that list retrieval cues may not be formed unless and until the $S$ finds this to be necessary. Except for clarifying this apparent conceptual similarity, there is no need to compare the present theory with Bower's comments on retroactive inhibition because, as Bower (1972) notes, his conceptualization is very similar to the hypothesis of response-set interference, except that the suppression of list markers replaces the notion of suppression by means of altered criteria for responding.

\section{INTERPRETATION OF EMPIRICAL PHENOMENA}

In this section we will consider the explanations offered by the present theory for unlearning phenomena which have been demonstrated in the A-B, A-C paradigm as well as the extension of the theory to proactive inhibition.

Within the A-C paradigm, it has been established that retroactive inhibition decreases as the degree of first-list learning increases (e.g., Briggs, 1957) and that retroactive inhibition increases as the degree of second-list learning increases (Barnes \& Underwood, 1959). The effect of first-list strength may be due to either of two factors depending upon the relative strength of the two lists. When the second list is stronger than the first, unlearning may decrease as first-list learning increases because more of the first-list responses intrude during second-list learning and, thus, more of the 
first-list responses are stored within the second-list memory unit. Support for this contention is provided in Experiment II by the finding that there is a significant tendency for strong items to intrude more often than weak items $[t(15)=2.87, p<.05]$. The mean proportion of strong items which intruded was 0.52 $(\mathrm{SD}=0.25)$; for weak items the mean proportion was $0.29(\mathrm{SD}=0.24)$.

When the first list is stronger than the second list, unlearning may also be decreased by the fact that the first-list memory unit remains dominant. If the first-list memory unit is more strongly associated with contextual cues than the second-list unit, $S$ may be able to retrieve the first-list memory unit directly. The effect of degree of second-list learning is attributed to the fact that the probability of retrieving the first-list memory unit decreases as the second-list memory unit becomes well integrated and increasingly dominant.

In addition to the present theory's ability to incorporate these findings, it also can explain two findings that Postman and Underwood (1973) cite as providing difficulties for current interference theory: (1) the failure to find a positive correlation between degree of negative transfer and degree of unlearning and (2) the failure to find a positive relationship between the strength of particular second-list pairs and the ability to recall the corresponding first-list pairs. Traditional interference theory leads to the expectation that negative transfer and unlearning should covary because interlist intrusions during second-list learning are assumed to produce both effects. According to the present theory, however, the occurrence of an intrusion could prevent the retrieval of the second-list response on that trial, thus contributing to negative transfer, but the intrusion would not produce unlearning of the first-list pair. On the other hand, negative transfer might be minimal if few intrusions occurred, while unlearning would remain high because of the inaccessibility of the first-list memory unit.

With regard to specific pairs, traditional interference theory leads to the expectation that the first-list pair which corresponds to a rapidly learned second-list pair should be more likely to be recalled because there were fewer opportunities for that first-list pair to intrude during second-list learning. The present theory suggests, however, that a second-list pair may be learned rapidly either because the first-list pair never intruded or because the intruding first-list pair produced minimal interference. Since the present theory holds that first-list pairs which never intruded will be inaccessible at recall, rapid learning of a second-list pair does not imply that the corresponding first-list pair should be retrievable.

One further phenomenon which occurs in the A-C paradigm and, thus, requires explanation is spontaneous recovery (Postman, Stark, \& Fraser, 1968; Postman, Stark, \& Henschel, 1969). The explanation offered for this effect is similar to that offered by Postman and his colleagues in the studies cited above. As the time interval between second-list learning and the retention test increases, the dominance of the second-list memory unit decreases. Consequently, the probability that $S$ will recover the first-list memory unit increases.

Finally, some consideration should be given to proactive inhibition. The most reasonable derivation from the present theory would attribute proactive inhibition to two factors: specific competition between responses associated to the same stimulus and output interference. First, for those stimuli for which the second-list memory unit contains both first- and second-list responses, specific competition may increase over time as memory for list membership decreases (McCrystal, 1970). Second, as the tendency to retrieve the second-list memory unit first decreases over time, output interference should begin to affect second-list responses as well as first-list responses.

In summary, the present theory suggests that interference effects at recall are primarily due to dominance relationships and competition effects that occur between lists. At the same time, however, the present theory suggests that the effect of these list-level processes on individual items depends upon item-specific intrusion processes.

\section{REFERENCES}

Barnes, J. M.. \& Underwood, B. J. "Fate" of first-list associations in transfer theory. Journal of Experimental Psychology, 1959, 58,97-105.

Bower, G. H. Stimulus-sampling theory of encoding variability. In A. W. Melton and E. Martin (Eds.), Coding processes in human memory. Washington, D.C: Winston, 1972.

Briggs, G. E. Retroactive inhibition as a function of degree of $O I$ and IL. Journal of Experimental Psychology, 1957, 53, 60-67.

Flores, L. M., \& Brown, S. C. Comparison of measures of output order in free recall. Behavior Research Methods \& Instrum entation, 1974,6, 385-388.

Hudson, R. L., \& Dunn, J. E. A major modification of the Bousfield (1966) measure of category clustering. Behavior Research Methods \& Instrum entation, 1969, 1, 110-111.

Keppel, G., \& Rauch, D. S. Unlearning as a function of second-list error instruction. Journal of Verbal Learning \& Verbal Behavior, 1966, 5, 50-58.

Martin, E. \& Mackay, S. A. A test of the list-differentiation hypothesis. American Journal of Psychology, 1970, 83, 311-321.

McCrystal, T. J. List differentiation as a function of time and test order. Journal of Experimental Psychology, 1970, 83, $220-223$.

McGovern, J. B. Extinction of associations in four transfer paradigms. Psychological Monographs, 1964, 78(16, Whole No. 593).

Paul, C., \& Silverstein, A. Relation of experimentally produced interlist intrusions to unlearning and retroactive inhibition. Journal of Experimental Psychology, 1968, 76, 480-485.

Pellegrino, J. W. Effects of intralist response formal similarity upon paired-associate transfer and retroactive inhibition. Journal of Experimental Psychology, 1972, 92, 134-142.

Petrich, J. A. Organization of recall in the retroactive inhibition paradigm. Journal of Verbal Learning \& Verbal Behavior, $1973,12,294-301$.

Popp, R., \& Voss, J. F. Acquisition, transfer, and retention of $S-R$, and $S-R_{2}$ associations as a function of $S-R_{1}, S-R_{2}$ pattern. Journal of Experimental Psychology, 1965, 70, 304-308.

Postman, L., Keppel, G., \& Stark, K. Unlearning as a function of the relationship between successive response classes. Journal of Experimental Psychology, 1965, 69, 111-118.

Postman, L., \& Parker, J. F. Maint enance of first-list associations 
during transfer. American Journal of Psychology, 1970, 83, 171-188.

Postman, L., \& Stark, K. Retroactive inhibition as a function of set during the interpolated task. Journal of Verbal Learning \& Verbal Behavior, 1963, 1, 304-311.

Postman, L., \& Stark, K. Role of response availability in transfer and interference. Journal of Experimental Psychology, 1969 79, 168-177.

Postman, L., Stark, K., \& Fraser, J, Temporal changes in interference. Journal of Verbal Learning \& Verbal Behavior, $1968,7,672-694$

Postman, L., Stark, K., \& Henschel, D. Conditions of recovery after unlearning. Journal of Experimental Psychology, 1969. 82(1, Part 2).

Postman, L.. \& Underwood, B. J. Critical issues in interference theory. Mernory \& Cognition, 1973, 1, 19-40.
Winograd, E. List differentiation as a function of frequency and retention interval. Journal of Experimental Psychology, 1968. 76(2, Part 2).

\section{NOTE}

1. The group median was used because attempts to divide items into equal-sized categories on an individual basis required a large number of arbitrary classifications for items with the same number of correct responses. Although the group procedure did not produce equal-sized categories per S, all Ss did contribute it ems to both categories.

(Received for publication December 17, 1973; revision received June $4,1974$. 